\title{
SATISFAÇÃO PROFISSIONAL DA EQUIPE DE ATENDIMENTO DOMICILIAR TERAPÊUTICO DE UMA UNIDADE DE REFERÊNCIAAO PORTADOR DE HIV/AIDS DO ESTADO DO PARÁ
}

Cristiane da Silva Rosa ${ }^{1}$, Aida Maris Peres², Mariluci Alves Maftum³

RESUMO: Pesquisa realizada em uma unidade de referência especializada no atendimento ao portador de HIV/AIDS no Estado do Pará. Objetivos: identificar fatores ambientais e psicossociais que podem influenciar no nível de satisfação profissional de uma equipe de Atendimento Domiciliar Terapêutico e conhecer as estratégias utilizadas pela equipe para a promoção da sua satisfação profissional. Participaram nove profissionais da equipe de Atendimento Domiciliar Terapêutico. Os dados foram coletados mediante entrevista com questões abertas. Para a análise dos dados foi utilizada a técnica de análise de conteúdo. Como principais resultados verificou-se um equilíbrio existente entre satisfação e insatisfação dos profissionais, bem como uma diversidade de fatores ambientais e psicossociais que influenciam o nível de satisfação dos profissionais. Considera-se necessário a adoção de medidas de prevenção e promoção da saúde da equipe, haja vista as peculiaridades de seu trabalho em que o sofrimento e a morte fazem parte da rotina diária desses trabalhadores, gerando estresse e outros problemas de saúde mental.

PALAVRAS-CHAVE: Satisfação no emprego; Saúde mental; Recursos humanos.

\section{PROFESSIONAL SATISFACTION OF ATHERAPEUTIC HOME CARE TEAM IN A SPECIALIZED HEALTH UNIT, REFERENCE ON THE HEALTH CARE FOR THE HIV/ AIDS PATIENTS IN PARÁ STATE}

\begin{abstract}
The study was developed in a specialized health unit that is reference on health care for HIV/AIDS patients in Pará State, Brazil. Objectives: to identify environmental and psychosocial factors that might influence the level of professional satisfaction of a Therapeutic Home Care team, and to know the strategies used by the team to promote this professional satisfaction. Nine professionals of the Therapeutic Home Care team joined the study. Data were gathered through interviews with open questions. Data analysis was developed by the content analysis technique. As main results, it was unveiled that there is a balance between satisfaction and dissatisfaction among the workers, as well as a diversity of environmental and psychosocial factors that influence the professional's level of satisfaction. The adoption of preventive and health promotion measures is necessary once suffering and death are routinely faced in their daily work, which may foster stress and other mental health problems.
\end{abstract}

KEYWORDS: Job satisfaction; Mental health; Human resources.

\section{SATISFACCIÓN PROFESIONAL DEL EQUIPO DE ATENCIÓN DOMICILIAR TERAPÉUTICA DE UNA UNIDAD DE REFERENCIAAL PORTADOR DE HIV/SIDA DEL ESTADO DE PARÁ}

RESUMEN: Investigación realizada en una unidad de referencia especializada en la atención al portador de HIV/SIDA en el Estado de Pará/BR. Objetivos: identificar factores ambientales y psicosociales que pueden influenciar en el nivel de satisfacción profesional de un equipo de Atención Domiciliar Terapéutico y conocer las estrategias utilizadas por el equipo para la promoción de su satisfacción profesional. Participaron nueve profesionales del equipo de Atención Domiciliar Terapéutico. Los datos fueron colectados por medio de entrevista con preguntas abiertas. Para el análisis de los datos fue utilizada la técnica de análisis de contenido. Como principales resultados se verificó un equilibrio existente entre satisfacción e insatisfacción de los profesionales, bien como una diversidad de factores ambientales y psicosociales que influencian el nivel de satisfacción de los profesionales. Se considera necesaria la adopción de medidas de prevención y promoción de la salud del equipo, en vista de las peculiaridades de su trabajo en que el sufrimiento y la muerte hacen parte de la rutina diaria de estos trabajadores, generando estrés y otros problemas de salud mental.

PALABRAS-CLAVE: Satisfacción en el trabajo; Salud mental; Recursos humanos.

\footnotetext{
${ }^{1}$ Terapeuta Ocupacional. Unidade de Referência em Doenças Infecto-parasitárias Especiais do Estado do Pará-URE-DIPE.

${ }^{2}$ Enfermeira. Doutora. Professor Adjunto do Departamento de Enfermagem e do Programa de Pós-Graduação Mestrado em Enfermagem. Universidade Federal do Paraná-UFPR. Membro do Grupo de Pesquisas em Políticas, Gestão e Práticas de Saúde-GPPGPS.

${ }^{3}$ Enfermeira. Doutora. Professor Adjunto do Departamento de Enfermagem e do Programa de Pós-Graduação Mestrado em EnfermagemUFPR. Vice-coordenadora do Núcleo de Estudos, Pesquisa e Extensão de Cuidado Humano em Enfermagem-NEPECHE-UFPR
}

Autor correspondente:

Cristiane da Silva Rosa

Tv. Djalma Dutra, 1000 - 66113-010 - Belém-PA

Recebido: 01/01/09

E-mail: cristirosa26@gmail.com

Aprovado: 10/05/09 


\section{INTRODUÇÃO}

Satisfação no trabalho constitui fenômeno complexo e de difícil definição, pois trata de estado subjetivo que varia de circunstâncias, de pessoa para pessoa e pode ainda variar ao longo do tempo para a mesma pessoa. A satisfação está sujeita à influência de forças internas e externas ao ambiente de trabalho, pode afetar a saúde física e mental do trabalhador e interferir em seu comportamento profissional e/ou social. Estudos ${ }^{(1-4)}$ demonstram a existência de íntima relação entre psicodinâmica do trabalho e saúde e fornecem uma série de estratégias que visam reduzir ao máximo os agravantes que poderiam intervir de modo negativo na saúde dos trabalhadores.

O profissional necessita propiciar aos usuários que procuram as unidades de saúde, elementos que contribuam para a promoção, resgate e manutenção da sua saúde. Entretanto, esses profissionais enfrentam situações rotineiras ambientais e psicossociais relacionadas ao seu ambiente de trabalho que podem repercutir diretamente na qualidade dos serviços prestados, inclusive lhes causar sentimentos de insatisfação profissional.

Ressalta-se que no Brasil poucos estudos abordam o tema satisfação profissional em trabalhadores da área da saúde. Existe, portanto, uma lacuna com relação a esta temática ${ }^{(5)}$. Assim, a realização desta pesquisa foi motivada por presenciar diariamente os esforços desempenhados por uma equipe de Atendimento Domiciliar Terapêutico-ADT de uma unidade de referência especializada para atender portadores de HIV/AIDS, que procurava contornar rotineiramente as situações adversas para cumprir seu papel terapêutico com esses pacientes.

O trabalho desempenhado pela equipe ao portador de HIV/AIDS na modalidade de ADT oferece vantagens para os doentes e seus familiares, colabora para evitar reinternações, diminui o risco de infecções hospitalares, aumenta o conforto dos pacientes e contribui para diminuir custos com internações.

O ADT é uma iniciativa do Ministério da Saúde, surgida no Brasil na década de 1990 e integra um conjunto de estratégias assistenciais, cujo objetivo é melhorar a qualidade de vida dos indivíduos portadores de HIV/AIDS. Conforme o preconizado pelo Manual de ADT, uma equipe multiprofissional deverá proporcionar assistência integral a estes usuários e seus familiares sem a necessidade de que os mesmos se desloquem ao centro de atendimento e assim, todos os atendimentos devem ser realizados no domicílio do paciente ${ }^{(6)}$. Este serviço está sustentado nas políticas de humanização e equivale ao atendimento prestado por um hospital.

Estima-se que no Estado do Pará existem aproximadamente 4.017 pessoas soropositivas notificadas. Para o atendimento hospitalar especializado, a rede é composta de 50 leitos, 40 em Belém e dez em quatro municípios no interior do Estado. Mesmo com a disposição de hospitais-dia e ADT, a demanda reprimida caracteriza deficiências na assistência ${ }^{(7)}$.

Nesta pesquisa os objetivos foram: identificar fatores ambientais e psicossociais que podem influenciar no nível de satisfação profissional de uma equipe de ADT e identificar estratégias que a equipe utiliza para a promoção da sua satisfação profissional.

\section{TRABALHO E SUAS RELAÇÕES COM O INDIVÍDUO}

O trabalho constitui uma das mais importantes maneiras do homem se posicionar como indivíduo único e é algo que complementa e confere sentido à sua vida. Também é considerado como um dos componentes da felicidade humana e, tem-se que a felicidade no trabalho é resultante da satisfação plena de necessidades psicossociais, do sentimento de prazer e do sentido de contribuição no exercício da atividade profissional ${ }^{(2)}$.

Estudos $^{(1-5)}$ indicam que a satisfação no trabalho exerce influência sobre o trabalhador; afeta sua saúde física e mental, atitudes, comportamento profissional com repercussões para a vida pessoal, familiar e para as organizações. O primeiro estudo sobre satisfação no trabalho foi realizado em 1920 e, desde então, diversos referenciais teóricos surgiram com o objetivo de elucidar tal temática ${ }^{(2)}$.

Nesta pesquisa utilizou-se como referenciais a Teoria da Psicopatologia e Psicodinâmica do Trabalho, que tem Dejours ${ }^{(2)}$ como seu principal autor e a Teoria de Locke. Ambas as teorias contemplam as relações entre satisfação no trabalho e saúde em que a satisfação no trabalho está relacionada ao prazer ou à felicidade no trabalho e a insatisfação está na origem do desprazer ou do sofrimento no trabalho.

A Teoria da Psicopatologia e Psicodinâmica do Trabalho tem pressupostos baseados na psicanálise e seu ponto positivo é realizar uma análise da psicodinâmica das situações que envolvem o trabalho. Destarte, o trabalho se torna perigoso para o aparelho psíquico a partir do momento em que se opõe à livre 
atividade na qual a liberdade para a organização do trabalho é limitada e se opõe aos desejos do trabalhador, gerando aumento da carga psíquica, provocando sofrimento no trabalho(2).

Neste contexto, o bem estar, levando-se em consideração a carga psíquica, decorre da existência de um sentido atribuído ao trabalho desenvolvido. A partir do momento em que o trabalho permite o alívio da carga psíquica, este passa a ser um instrumento de equilíbrio e fonte de prazer para o trabalhador e, no contrário, dá origem a sofrimento e patologia. Esse sofrimento representa uma vivência subjetiva intermediária entre transtorno mental e o bem estar psíquico, porém haverá luta entre as forças que impulsionam o indivíduo em direção ao transtorno mental.

A insatisfação no trabalho pode se originar em decorrência de sentimentos de indignidade pela obrigação de realizar uma tarefa desinteressante, sem significado, desconhecer o que realmente significa seu trabalho no conjunto das atividades da instituição, desqualificação, questões salariais, como também pela valorização do trabalho, em aspectos como responsabilidade, risco ou conhecimentos necessários ${ }^{(2)}$.

A teoria apresentada por Locke considera a satisfação no trabalho o resultado entre o que sujeito deseja de seu trabalho e o que ele percebe que está obtendo. Já a insatisfação constitui estado emocional não prazeroso, resultante da avaliação do trabalho que ignora, frustra ou nega valores do indivíduo. Ressaltase que satisfação e insatisfação no trabalho não são fenômenos distintos, e sim, situações opostas de um mesmo fenômeno. Trata-se de um estado emocional que se manifesta na forma de alegria (satisfação) ou sofrimento (insatisfação). O trabalho é uma interação complexa de tarefas, papéis, responsabilidades, relações, incentivos e recompensas em determinado contexto físico e social ${ }^{(2)}$.

Embora haja diferenças individuais significativas em relação aos anseios dos trabalhadores com seus trabalhos, existem grandes semelhanças entre os fatores que causam satisfação. Esses fatores estão relacionados a questões como pagamento, promoção, reconhecimento, condições e tipo de trabalho, ambiente, colegas, supervisão, gerenciamento, empresa/organização. Existem outros determinantes que influenciam na satisfação individual do trabalho como diferenças de personalidade e valores que a pessoa atribui ao trabalho(2).

Ressalta-se a ocorrência de melhor qualidade da saúde física em indivíduos satisfeitos e, como consequência, o aumento da longevidade. Para indivíduos insatisfeitos há maior risco para a ocorrência de problemas de saúde: fadiga, dificuldade respiratória, dor de cabeça, perda de apetite, aumento do colesterol, problemas digestivos, doença cardíaca aterosclerótica e dores musculares. A justificativa para tais sintomas estaria no fato de que a satisfação/insatisfação é um estado emocional, havendo respostas físicas involuntárias dependendo do teor da emoção ${ }^{(2)}$.

Com relação à saúde mental, ao contrário do que se pode supor, a organização do trabalho não cria doenças mentais específicas. Os surtos psicóticos e a formação das neuroses dependem da estrutura da personalidade que a pessoa desenvolve desde o início da sua vida, porém, a organização do trabalho pode favorecer as descompensações psiconeuróticas. Contudo, o trabalho também é enfocado como regulador social ${ }^{(1)}$, importante para a subjetividade humana e manutenção da saúde mental.

Os próprios trabalhadores têm condições de reconhecer as situações de risco do trabalho para a sua saúde, entretanto se mantém na submissão a essas condições ${ }^{(4)}$. Ouve-se dizer comumente que possuir um emprego, sem considerar o tipo e suas condições, é suficiente para se considerar uma pessoa de sorte. Essa submissão consciente a condições inadequadas, particularmente no que diz respeito ao ritmo de trabalho e às pressões cotidianas, parece que está se tornando um fator adicional de sofrimento psíquico que merece uma atenção especial por parte dos profissionais da área de saúde mental e trabalho.

\section{METODOLOGIA}

Esta pesquisa foi realizada no ano de 2007, em uma unidade de referência especializada em atendimento ao portador de HIV/AIDS do Estado do Pará.

O projeto foi aprovado pelo Comitê de Ética e Pesquisa da Fundação Pública Hospital de Clínicas Gaspar Vianna sob o protocolo $n^{\circ} 081 / 07$. Os sujeitos assinaram o Termo de Consentimento Livre e Esclarecido seguindo as recomendações da Resolução n 196/96 do Conselho Nacional de Saúde ${ }^{(8)}$.

Os sujeitos desta pesquisa foram nove profissionais da equipe de ADT: médico, psicólogo, assistente social, enfermeira, nutricionista, fisioterapeuta, terapeuta ocupacional, técnico de enfermagem e motorista. O critério de inclusão foi pertencer à equipe de trabalho no período vespertino e aceitar participar da coleta de dados. Foram excluídos 
os profissionais que trabalham na equipe do período matutino, aqueles que se recusaram participar desta pesquisa e os que não integram o programa de ADT.

A coleta de dados foi realizada por meio de entrevista com perguntas abertas a respeito dos fatores que influenciam na satisfação profissional dos trabalhadores e sobre a satisfação profissional com o trabalho desenvolvido na equipe de ADT.

A análise dos dados obtidos nas entrevistas foi realizada mediante a técnica de análise de conteúdo proposta por Bardin ${ }^{(9)}$ e organizados em quatro categorias temáticas: O programa de Atendimento Domiciliar Terapêutico; Fatores de satisfação e insatisfação profissional da equipe de ADT; Condições ambientais e psicossociais de trabalho da equipe de ADT; e Estratégias adotadas pela equipe de ADT para aumentar/melhorar a satisfação profissional.

\section{APRESENTAÇÃO E DISCUSSÃO DOS DADOS}

\section{O programa de atendimento domiciliar terapêutico}

O serviço de ADT foi implantado em 2003 e até a época da coleta dos dados desta pesquisa já havia atendido 50 pacientes em diversos bairros de Belém. Existem pacientes que estão no programa desde sua implantação e isso permite que os leitos hospitalares fiquem liberados para situações de emergências.

Os critérios para inclusão/exclusão do paciente no programa abrangem aspectos socioeconômicos, psicossociais, clínicos, dificuldades de deambulação até a unidade de saúde para realização de seu tratamento. É considerado, em especial, a baixa condição socioeconômica do paciente. O paciente geralmente é encaminhado por outros profissionais ou o familiar procura o serviço. Inicialmente é realizada uma avaliação pela equipe e depois da primeira visita cada profissional emite parecer e juntos decidem se o indivíduo apresenta ou não critérios para ser atendido pelo programa.

As pessoas portadoras do HIV/Aids vivem sob forte estigma social, e geralmente são vistas como se tivessem cometido um erro ou moralmente repulsivas. Diante disso, muitos pacientes relutam em contar a seus familiares e amigos que são portadores do vírus ou que têm a doença. Além disso, muitos carregam a sensação de culpa pela possibilidade de terem infectado outras pessoas e chegam a acreditar que são merecedoras de terem sido contaminadas pelo HIV. Agravando tal situação, nossa sociedade ainda vê a Aids como doença da promiscuidade o que gera preconceito social e portanto uma doença que provoca profundo estresse negativo ${ }^{(10)}$.

Como o vírus ataca o sistema imunológico, o indivíduo é obrigado a conviver diariamente com o sentimento de que pode contrair uma doença oportunista a qualquer momento. Além disso, vários medos rondam o imaginário do paciente como, por exemplo, o relacionado à morte de outras pessoas portadoras do vírus, dos efeitos colaterais provocados pelo uso contínuo dos antiretrovirais. Assim, muitos não contam aos familiares, amigos ou empregadores que são HIV positivos ou que tem Aids e o único apoio desses indivíduos, por vezes, é a equipe profissional que presta 0 atendimento na unidade de referência ${ }^{(10)}$.

O trabalho com pacientes que apresentam toda a problemática citada anteriormente envolve constantemente dor, sofrimento e morte. Se por um lado os profissionais de ADT possuem o papel de aliviar a dor e o sofrimento do portador de HIV/AIDS e seus familiares, de outro, fazem parte de um serviço que passa por constantes modificações relacionadas à organização do trabalho e assim, são submetidos às mesmas regras impostas aos demais trabalhadores de qualquer empresa.

\section{Fatores de satisfação e insatisfação profissional da equipe de ADT}

Em relação ao trabalho desenvolvido na equipe de ADT, uma parcela dos sujeitos referiu que estava satisfeita e considerou como motivos a relação com o paciente em seu domicílio, a troca de saberes entre profissional-paciente-familiar e comunidade, a possibilidade de prestar assistência clínica, emocional e social, além da coesão da equipe, cujo ponto de convergência é o paciente.

Outra parcela dos profissionais referiu insatisfação com o trabalho desenvolvido e ressaltou que pelo fato do serviço ter sido estruturado de forma improvisada, o mesmo estava funcionando sob condições precárias de estrutura física, com falta de materiais, equipamentos e medicamentos. Também foi destacado pelos sujeitos que outros profissionais que atuam na unidade de saúde, por não entender e desvalorizar o trabalho da equipe de ADT, tinham atitudes que influenciavam o usuário a desacreditar no serviço. Houve uma parcela menor que relatou existir momentos de satisfação e de insatisfação, dependendo do aspecto considerado e em concordância 
com as respostas referidas anteriormente.

Essa divergência de respostas, na quais a subjetividade se faz presente, confirma o que autores teorizam sobre o conceito de satisfação profissional. Porquanto, satisfação no trabalho é definida como um estado emocional resultante da interação do profissional, suas características pessoais, valores e expectativas com o ambiente e a organização do trabalho. Por se tratar de conceito subjetivo e variável conforme a circunstância, convém esclarecer que os dados desta pesquisa foram coletados em momento de mudança de gestão da unidade, em que várias transformações do ambiente de trabalho estavam na eminência de acontecer e os profissionais estavam com baixa tolerância sobre as diversas tentativas frustradas de conseguir melhorias em seu ambiente de trabalho( ${ }^{(5)}$.

\section{Condições ambientais e psicossociais de trabalho da equipe de ADT}

Os sujeitos explicitaram a inexistência de condições ambientais e psicossociais para o desempenho do seu trabalho, como falta de espaço físico definido dentro da unidade de saúde, equipamentos sucateados, falta de um automóvel próprio para transportar os profissionais até a residência dos usuários. Referiram como condições psicossociais a desvalorização do serviço pelos outros profissionais da unidade, desinteresse da gerência em priorizar o programa, excessiva demanda de trabalho, as situações psicossociais do próprio usuário e que esses aspectos acarretam sobrecarga intelectual e emocional prejudicando a saúde mental deles.

Destaca-se que o problema relacionado ao ambiente físico estende-se também aos outros serviços da unidade. Os funcionários estavam, naquele momento, vivenciando a expectativa de mudança de prédio e isso, de acordo com eles, gerava ansiedade com relação a possibilidade de melhorias de condições ambientais.

Foi constatada a inexistência de programa específico de saúde do trabalhador para o atendimento da equipe da unidade com enfoque na prevenção e promoção de sua saúde. Assim, eles não dispõem de um espaço para discutir situações relacionadas aos pacientes, ao atendimento dessa clientela com características de saúde-doença específica e à organização de seu ambiente de trabalho.

Entende-se por ambiente psicossocial tudo que envolve a organização do trabalho, incluindo as relações sociais dos componentes de uma instituição.
Deste modo, a escuta do trabalhador é importante para identificar fatores causadores de insatisfação como para avaliar possibilidades de redução dos riscos que podem levar ao adoecimento. Porquanto, escutar ou ouvir reflexivamente o outro é uma ação indispensável às relações de ajuda e terapêutica ${ }^{(11-13)}$.

Os sujeitos atribuem importância aos sentimentos de insatisfação relacionado à falta de reconhecimento pelo trabalho desenvolvido perante os outros funcionários da unidade. No estudo ${ }^{(12)}$ é ressaltado que na medida em que realiza seu trabalho, independente do resultado, o profissional deseja o reconhecimento, sendo que este não deveria ocorrer apenas devido à sua contribuição, mas que a contribuição deve ser julgada pelos outros como positiva e necessária.

Com relação a melhoria das condições de trabalho, os sujeitos referiram urgência na adequação e ampliação do espaço físico, com acessibilidade aos portadores de deficiência e definição de uma área física dentro da própria unidade para alocar o programa de ADT. Enfatizaram que nesse espaço deveria haver reuniões para estudos de casos, apresentação do programa para os outros profissionais e usuários, veículo próprio para transportar a equipe, materiais e equipamentos específicos de cada categoria profissional. Referiram também necessidade de reconhecimento do programa como um serviço essencial, tanto por parte dos gestores dos serviços de atenção básica, como pela gerência da própria unidade.

Os sujeitos apontaram a necessidade de criação de grupos de auto-ajuda, escuta, terapia ocupacional tanto para os trabalhadores como para os usuários, implementação de programa de educação permanente e atenção para os processos de trabalho com reorganização de carga horária. Também explicitaram que os outros profissionais da unidade deveriam participar das discussões da equipe, para que tivessem oportunidade de conhecer e vivenciar as especificidades do programa.

Ressalta-se que o reconhecimento do trabalho desenvolvido pela pessoa constitui

retribuição simbólica que pode tomar sentido em relação aos anseios subjetivos quanto a realização de si. $\mathrm{Na}$ dinâmica de construção/fortalecimento da identidade, 0 reconhecimento do fazer vem necessariamente em primeiro lugar $^{(12: 844)}$.

Estratégias adotadas pela equipe de ADT para aumentar/melhorar a satisfação profissional 
Em situações nas quais existe sofrimento provocado pelo ambiente de trabalho, é comum que o trabalhador adote estratégias defensivas com a finalidade de se proteger de adoecimento. Assim, os sujeitos relataram estratégia que adotam para aumentar/ melhorar a satisfação profissional, como procurar exercer sua cidadania, investir na capacitação profissional através de cursos, oficinas de aperfeiçoamento, reflexão em equipe acerca das dificuldades vivenciadas, elaboração de estratégias de melhoramento do serviço, cobranças à gerência, manutenção de boa interação com os membros da equipe, além do envolvimento com outros serviços da unidade. Uma minoria referiu que realiza atividade física.

O exercício de questionar e refletir sobre suas práticas profissionais é importante no sentido de que o mundo do trabalho encontra-se sob um delicado processo de reestruturação, havendo com isso necessidade de mudança do perfil dos trabalhadores. Nesse perfil, devem se fazer presentes ou serem estimulados nos trabalhadores competências como valorização da polivalência, comportamento organizacional, qualificação técnica, participação criadora, mobilização da subjetividade, capacidade de diagnosticar e de decidir(13).

A reflexão sobre suas práticas que a equipe de ADT está fazendo, somada às condições adversas de trabalho em que estão submetidos, pode estimular o potencial criador dos profissionais, no sentido de encontrar alternativas que possibilitem minimizar os agentes causadores de insatisfação. Essa atitude ativa e reflexiva em situações de trabalho nas quais exista grande demanda e limitada capacidade de decisão, levam ao esgotamento do trabalhador. Ao contrário, condições com alto nível de demandas, porém que oferecem aos trabalhadores o poder de tomar decisões, induzem à menor taxa de esgotamento ${ }^{(12)}$.

Os profissionais da equipe de ADT foram unânimes em afirmar que a qualidade do atendimento ao usuário pode ser afetada pela insatisfação de trabalho da equipe, pois condições insatisfatórias de trabalho fazem com que a agenda semanal das visitas domiciliares não seja cumprida e se tornem aquém das necessidades destes, o que pode influenciar na qualidade dos atendimentos realizados. Explicitaram que existem usuários com sequelas irreversíveis decorrentes de doenças oportunistas e que assim, dependem dessa modalidade de tratamento para continuar sobrevivendo.

Os sujeitos relataram que os sentimentos originados a partir da sua prática ocorrem conforme o profissional consegue ou não realizar seu trabalho. Quando conseguem, apesar das condições adversas existentes, os sentimentos mais comentados foram: alegria, satisfação, ânimo, sensação de crescimento profissional. Por outro lado, quando não conseguem os sentimentos são de impotência, tristeza, frustração, decepção, angústia, insatisfação, medo, revolta, cansaço físico e mental, desânimo, desmotivação, desejo de mudar de trabalho, raiva.

É importante identificar esses sentimentos, bem como as situações do ambiente de trabalho suscitadoras dos sentimentos negativos as quais devem ser sanadas, pois existe uma dificuldade em correlacionar distúrbios psíquicos e ambiente de trabalho, visto estarem diretamente relacionados com individualidade e subjetividade do trabalhador e sensibilidade do profissional que investiga e avalia o ambiente e a organização do trabalho ${ }^{(14)}$.

Apesar das dificuldades encontradas para a realização de suas práticas profissionais a equipe se mantém na unidade. Os entrevistados referiram que é devido ao amor à profissão, ao vínculo estabelecido entre os membros da equipe e com os usuários, à necessidade de trabalhar e à esperança de que a atual situação se modifique.

Destaca-se que a coesão da equipe e vínculo com os pacientes representa um forte fator de proteção à saúde dos profissionais ${ }^{(12)}$. Em outra pesquisa ${ }^{(13)}$ encontrou-se que os relacionamentos interpessoais, internos e externos, são importantes para a satisfação com o trabalho, sendo que há fortes evidências de que relações sociais conflituosas podem afetar a saúde, aumentar a carga de trabalho, havendo consequente desgaste físico e mental.

\section{CONCLUSÃO}

Este estudo evidenciou um equilíbrio quanto à existência de satisfação e insatisfação dos profissionais atuantes no programa de ADT, bem como uma variedade de fatores ambientais e psicossociais que influenciam o nível de satisfação.

Além das questões ambientais, como falta de espaço físico para alocar o programa, meio de transporte, falta de materiais e equipamentos, há também o sentimento de desvalorização e falta de reconhecimento dos outros profissionais que trabalham na unidade de saúde. Entretanto, a equipe destacou como ponto positivo o entrosamento existente entre os membros da equipe de $\mathrm{ADT}$, que por meio deste 
relacionamento eles se fortificam, se unem coletivamente na tentativa de modificar e melhorar as suas condições de trabalho.

Considera-se importante que a gestão da unidade invista em medidas de prevenção e promoção da saúde da equipe de ADT, haja vista as peculiaridades de seu trabalho com pessoas com doença crônica em que o sofrimento e a morte fazem parte da rotina diária da prática profissional desses trabalhadores, gerando estresse e outros problemas de saúde mental. Do mesmo modo, é necessária a avaliação constante das condições de trabalho propiciadas pelo ambiente.

Essa pesquisa demonstrou a existência de um campo vasto e ainda pouco estudado pelos próprios profissionais da saúde que é a prevenção do adoecimento mental relacionado com as condições físicas e psicossociais de trabalho.

\section{REFERÊNCIAS}

1. Capitão CG, Heloani JR. Saúde mental e psicologia do trabalho. Perspectiva. 2003 Abr/Jun; 17(2):59-78.

2. Martinez MC, Paraguay AIBB. Satisfação e saúde no trabalho: aspectos conceituais e metodológicos. Cad Psicol Social Trab. 2003 Dez; 6(1):59-78.

3. Latorre MRDO, Martinez MC, Paraguay AIBB. Relação entre satisfação com aspectos psicossociais e saúde dos trabalhadores. Rev Saúde Públ. 2004 Fev; 38(1):34-55.

4. Sato L, Bernardo MH. Saúde Mental e trabalho: os problemas que persistem. Ciênc Saúde Col. 2005 Out/ Dez; 10(4):23-46.

5. Rebouças DR, Legay LF, Abelha L. Satisfação com o trabalho e impacto causado nos profissionais de serviço de saúde mental. Rev Saúde Públ. 2007 Jan;41(2):244-50.

6. Ministério da Saúde (BR). Manual de assistência domiciliar terapêutica (HIV/AIDS). Brasília: Ministério da Saúde; 1999.

7. Secretaria Estadual de Saúde do Pará. DST/AIDS. [Acesso em 2009 Mai 15] Disponível: http:// portal.sespa.pa.gov.br/portal/page?_pageid=73,36424 \&_dad=portal\&_schema=PORTALpagina=dstaids.html.

8. Ministério da Saúde (BR). Conselho Nacional de Saúde. Diretrizes e normas regulamentadoras de pesquisa envolvendo seres humanos. Resolução n. 196, de 10 de outubro de 1996. Brasília; 1996.
9. Bardin L. Análise de conteúdo. Lisboa: Edições 70; 1977.

10. Gutterman L. Infecção por HIV e AIDS. In: Early MB, Pedretti LW. Terapia ocupacional: capacidades para as disfunções físicas. $5^{\text {a }}$ ed. São Paulo: Roca; 2005. p.106876.

11. Roehrs H, Maftum MA, Stefanelli MC. A comunicação terapêutica sustentando a relação interpessoal entre adolescente e professor do ensino fundamental . Online Brazilian Journal [periódico na Internet]. 2007;6(3) [acesso em 2008 Out 30]. Disponível: http:// www.uff.brobjnnursing/index.php/nursing/ articleviewArticle/j.1676-4285.2007.1

12. Palácios M, Duarte F, Câmara VM. Trabalho e sofrimento psíquico de caixas de agências bancárias na cidade do Rio de Janeiro. Cad Saúde Públ. 2002 Mai/ Jun;18(3):843-51.

13. Gasparini MG, Barreto SM, Assunção AÁ. Prevalência de transtornos mentais comuns em professores da rede municipal de Belo Horizonte, Minas Gerais, Brasil. Cad Saúde Públ. 2006 Dez;22(12):2679-91.

14. Rocha LE, Vieira MG, Glyna DMR, Batista ML. Saúde Mental e trabalho: uma reflexão sobre o nexo com o trabalho e o diagnóstico, com base na prática. Cad Saúde Públ. 2001 Mai/Jun;17(3):01-13. 